\title{
The Long-Term Outcomes for Patients with Budd-Chiari Syndrome Caused by Behcet's Disease: A Case Series on the Results, from Cirrhosis to Death
}

\author{
Dondu Uskudar Cansu, ${ }^{1,}$ Tuncer Temel, ${ }^{2}$ Adem Erturk, ${ }^{1}$ Timucin Kasifoglu, ${ }^{1}$ Berat Acu, ${ }^{3}$ and Cengiz \\ Korkmaz $^{1}$ \\ ${ }^{1}$ Division of Rheumatology, Department of Internal Medicine, Eskisehir Osmangazi University, Eskisehir, Turkey \\ ${ }^{2}$ Division of Gastroenterology, Department of Internal Medicine, Eskisehir Osmangazi University, Eskisehir, Turkey \\ ${ }^{3}$ Department of Radiology, Eskisehir Osmangazi University, Eskisehir, Turkey \\ "Corresponding author: Dondu Uskudar Cansu, Department of Rheumatology, School of Medicine, Eskisehir Osmangazi University Eskisehir, Turkey. Tel: +90-2222392979-2931, \\ Fax: +90-2222393772, E-mail: ducansu@hotmail.com
}

Received 2015 August 15; Revised 2016 August 16; Accepted 2016 August 17.

\begin{abstract}
Background: Budd-Chiari syndrome, which is a rare complication of Behcet's disease, carries a high mortality rate.

Objectives: The aim of the study was to present our long-term follow up experience with patients suffering from Budd-Chiari syndrome due to Behcet's disease.

Methods: The records of 402 patients with Behcet's disease were evaluated retrospectively. To facilitate detection of the long-term complications caused by Budd-Chiari syndrome, the patients were evaluated via physical examinations, laboratory tests, imaging modalities, and endoscopy results.

Results: The data for 402 patients diagnosed with Behcet's disease, who were followed up at our hospital over 16 years, were analyzed retrospectively. Five of these 402 patients (1.2\%) were diagnosed as having Budd-Chiari syndrome. The patients with Budd-Chiari syndrome were aged between 23 and 54, and all five were male. The interval between the onset of Behcet's disease and the development of Budd-Chiari syndrome ranged from 1 to 8 years. All the patients had combined venous occlusion (affecting the hepatic vein and inferior vena cava). Portal venous thrombosis was detected in only one patient (Case 1), who died 1 month after the diagnosis of Budd-Chiari syndrome. The survival time for the other four patients after the diagnosis of Budd-Chiari syndrome ranged from 4 to 16 years. During the long-term follow-up, hepatic masses were detected via radiological surveillance in Case 3 (in the form of large regenerative nodules) and Case 4 (nodular regenerative hyperplasia and cirrhosis).

Conclusions: In our study, portal venous thrombosis was detected in the patient who died during the acute period only. A study including large numbers of Budd-Chiari-syndrome patients with Behcet's disease and portal venous thrombosis would be helpful to determine the prognostic significance of portal venous thrombosis in Budd-Chiari-syndrome patients with Behcet's disease. In addition, patients should be monitored regularly for the development of hepatic masses via a long-term surveillance program.
\end{abstract}

Keywords: Behcet's Disease, Budd-Chiari Syndrome, Portal Vein, Thrombosis, Prognosis, Cirrhosis

\section{Background}

Behçet's disease (BD) is the systemic vasculitis of small and large vessels, which affects both veins and arteries. The clinical features of BD indicate that there are distinct geographical differences; while vascular involvement is detected in $40 \%$ of patients in the Middle East, the percentage of vascular involvement is 5\% -10\% in the Far East (Japan) (1, 2). Thrombosis of the major veins such as the superior and inferior vena cava can be seen occasionally. Budd-Chiari syndrome (BCS) is a rare occurrence in the course of BD and carries a high mortality rate. BCS is caused by an outflow obstruction in any part of the hepatic venous system, from the small hepatic veins (HV) to the inferior vena cava (IVC) (3). The etiology of BCS also varies according to geography. While thrombosis is more prominent in the West, webs are prominent in the East and in Japan. BD is a more prominent etiological factor in Turkey than in other countries (4). Although there are studies that demonstrate the prognostic significance of the portal vein (PV), HV, and IVC in $\mathrm{BCS}$, there are no data in the literature explicating the prognosis and long-term outcomes for BCS that is secondary to $\mathrm{BD}(5)$. 


\section{Objectives}

In this retrospective study, we aimed to present the long-term follow-up outcomes for patients with BCS due to BD through clinical findings, laboratory test results, and the determination of vascular involvement.

\section{Methods}

The data for 402 patients diagnosed with BD (according to the diagnostic criteria for $\mathrm{BD}$ given by the international study group in 1990), who received regular follow ups at the rheumatology department at Eskisehir Osmangazi university medical faculty between 1998 and 2014, were evaluated retrospectively (6). Of the 402 patients with BD, 96 (23.9\%) had vascular involvement and five (1.2\%) were diagnosed as having BCS.

Doppler ultrasound (DUSG) and computed tomography (CT) were used as the first-line imaging modalities for the diagnosis of BCS. Vascular involvement sites causative of BCS were recorded. DUSG and magnetic resonance imaging (MR) or CT (for patients for whom MR was not suitable) were the preferred techniques for the long-term follow up of the patients. Ultrasonography-guided (USG) liver mass biopsies were performed on the patients in whom mass lesions were detected during surveillance (Cases 3 and 4 ).

The full biochemistry results, complete blood counts, erythrocyte sedimentation rates (ESR), and C-reactive protein (CRP) results for the patients were determined at the initial presentation stage and throughout the follow up. The congenital (protein C [PC], protein S [PS], and antithrombin III [ATIII] levels, and factor V Leiden mutations) and acquired causes (anticardiolipin and lupus antibodies) of thrombophilia were evaluated in all patients initially, at the time of BCS diagnosis. If the levels of PC, PS, and AT III were below the normal range, the study was repeated twice - at both 6 months and 1 year after the initial diagnosis (Cases 2, 3, 4, and 5).

The activity of the BD at the time of BCS diagnosis was determined using the European BD activity index (7).

For the detection of long-term complications, the patients were monitored via physical examinations, laboratory investigations, imaging modalities, and endoscopies.

\section{Results}

\subsection{Demographics and Clinical Findings}

The patients were aged between 23 and 54, and all five were male. The duration of $\mathrm{BD}$ in the patients ranged from 1 to 24 years (median: 12.4 years). The interval between the onset of $\mathrm{BD}$ and the development of BCS ranged from 1 to 8 years (mean: 3.8 years). The diagnostic age for BCS ranged from 21 to 38 years.

All the patients had genital ulcers, deep vein thrombosis (DVT), superficial thrombophlebitis (STP), and skin involvement at the time of diagnosis. No ocular involvement was detected. The patients presented with hepatomegaly, abdominal pain, ascites, and mild or moderate hyperbilirubinemia, leading to a diagnosis of BCS. The diagnoses of BD and BCS were made simultaneously in two of the patients (Cases 1 and 4 ). A family history was determined in Cases 3 and 4 .

\subsection{Laboratory Findings}

All the patients except one presented with severely increased serum aspartate aminotransferase (AST) and alanine aminotransferase (ALT) levels. The albumin levels of four of the patients were lower than usual. ESR levels were increased in three of the patients only, but CRP levels were elevated in all the patients. In terms of potential underlying thrombophilic conditions apart from BD, PC deficiency was detected in four of the patients. In addition to PC deficiency, factor V Leiden mutation was determined in Case 2.

\subsection{Vascular Involvement Sites and BCS Development}

Three types of venous outflow obstructions were recorded (Cases 1 and 5: involvement of $3 \mathrm{HVs}$ and the VCI; Cases 3 and 4: involvement of 2 HVs and the VCl; Case 2: involvement of $1 \mathrm{HV}$ and the VCI). In Case 1 , in addition to the involvement of $3 \mathrm{HVs}$ and the VCI, intra-cardiac thrombus and pulmonary embolism were detected. Portal venous thrombosis (PVT) was detected in only one of the patients (the detailed characteristics of the patients are given in Table 1).

\subsection{Long-Term Follow-Up Results for BCS in BD Patients}

One of the patients with BCS died during the acute period due to acute liver failure (ALF). The survival time for the other four patients after BCS diagnosis ranged from 4 to 16 years. Hepatic encephalopathy developed 4 months after the initial diagnosis of BCS in Case 4. Hepatic masses were determined by radiological surveillance in Case 3 (Figure 1) and Case 4 (11 and 4 years after the initial diagnosis of $\mathrm{BCS}$, respectively). Liver-mass biopsies revealed there to be large regeneration nodules (Case 3; Figure 2) and cirrhosis (Case 4). During endoscopic surveillance for the evolution of esophago-gastric varices, esophageal varices were determined in Case 4 only. With the exception of the patient in Case 1, who died during the acute period, all of the patients are still alive. In Case 4 , the patient was given a pathological diagnosis of Child-Pugh stage A cirrhosis. 
Table 1. Clinical Features, Laboratory Findings, Vascular Involvement Sites, and Outcomes for BCS Patients with BD

\begin{tabular}{|c|c|c|c|c|c|}
\hline & Case 1 & Case 2 & Case 3 & Case 4 & Case 5 \\
\hline \multicolumn{6}{|l|}{ Clinical findings } \\
\hline Age/Gender & $23 / \mathrm{M}$ & $54 / \mathrm{M}$ & $34 / \mathrm{M}$ & $33 / \mathrm{M}$ & 29/M \\
\hline Onset age of BD & 22 & 30 & 20 & 19 & 20 \\
\hline Diagnostic age of BD & 23 & 37 & 22 & 21 & 23 \\
\hline Diagnostic age of BCS & 23 & 38 & 23 & 21 & 25 \\
\hline Duration of BD & 1 year & 24 years & 14 years & 14 years & 9 years \\
\hline $\begin{array}{l}\text { Onset of BCS after the diagnosis of } \\
\text { BD }\end{array}$ & 1 year & 8 years & 3 years & 2 years & 5 years \\
\hline Aphtous ulcer & + & + & + & + & + \\
\hline Genital ulcers & + & + & + & + & + \\
\hline Eye involvement & - & - & - & - & - \\
\hline DVT/STP & $+/+$ & $+/+$ & $+1+$ & $+1+$ & $+1+$ \\
\hline PAA/Intracardiac thrombosis & $-1+$ & + & + & + & + \\
\hline Pathergy test & - & - & - & + & - \\
\hline Skin involvement & + & + & + & + & + \\
\hline HLA B5 & - & + & - & - & - \\
\hline Family history of BD & - & - & + & + & - \\
\hline \multicolumn{6}{|l|}{ Laboratory findings } \\
\hline Thrombophilic condition & - & FV leiden (+) PC $\downarrow$ & $\mathrm{PC} \downarrow$ & $\mathrm{PC} \downarrow$ & $\mathrm{PC} \downarrow$ \\
\hline AST(first/last) (IU/L) & $1421 /-$ & $80 / 22$ & $1131 / 22$ & $1278 / 31$ & $516 / 46$ \\
\hline $\operatorname{ALT}($ first/last) (IU/L) & $128 /-$ & $106 / 32$ & $608 / 14$ & $2057 / 16$ & $584 / 19$ \\
\hline GGT(first/last) (IU/L) & - & - & $491 / 290$ & $96 / 64$ & $64 / 49$ \\
\hline Albumin (first/last) (g/dL) & $2.77-$ & $4 / 4.7$ & $2.8 / 4.7$ & $2.7 / 3.1$ & $2.5 / 4$ \\
\hline ESR (first/last) $(\mathrm{mm} / \mathrm{h})$ & - & $33 / 17$ & $54 /-$ & $2 / 11$ & $28 / 11$ \\
\hline $\mathrm{CRP}$ (first/last) (mg/dL) & - & $3.68 / 0.1$ & $3.8 /-$ & $3.08 / 0.34$ & $7.19 / 1.33$ \\
\hline Child-Pugh (first/last) & - & $\mathrm{C} / \mathrm{A}, 10 / 5$ & $\mathrm{C} / \mathrm{A}, 10 / 5$ & $\mathrm{C} / \mathrm{A}, 14 / 5$ & $\mathrm{~B} / \mathrm{A}, 9 / 5$ \\
\hline BD activity index & 5 & 4 & 6 & 5 & 6 \\
\hline \multicolumn{6}{|l|}{$\begin{array}{l}\text { Vascular involvement sites and } \\
\text { outcomes }\end{array}$} \\
\hline Vascular involvement site & $3 \mathrm{HV} / \mathrm{IVC}$ & 1HV/IVC & 2HV/IVC & 2HV/IVC & 3HV/IVC \\
\hline PV thrombosis & + & - & - & - & - \\
\hline Life time after diagnosis of BCS & 1 month & 16 years & 11 years & 10 years & 4 years \\
\hline $\begin{array}{l}\text { Complications of BCS } \\
\text { cirrhosis/HEP/Varices }\end{array}$ & Exitus at acute period & Off follow up & $H$ & $+|+|+$ & $-H$ \\
\hline Long-term outcomes & Exitus in acute period & Off follow-up (alive) & LRNs (alive) & NRH, Cirrhosis (alive) & Irregular liver pattern (alive) \\
\hline
\end{tabular}

Abbreviations: BD, Behcet's disease; BCS, Budd-Chiari syndrome; M, male; DVT, deep vein thrombosis; STP, superficial thrombophlebitis; PAA, pulmonary artery aneurysms; AST, aspartate aminotransferases (normal range 7 - 39 U/L); ALT, alanine aminotransferases (normal range 2 - 40 U/L); GGT, gamaglutamil transferases (normal range 8 - 50 348U/L); ESR, erythrocyte sedimentation rate; CRP, C-reactive protein (normal range 0 - $0.5 \mathrm{mg} / \mathrm{dL}$ ); FV, factor V; PC, protein C; PV, portal vein; HEP,hepatic encephalopathy; HV, hepatic vein; IVC, inferior vena cava; LRNs, large regenerative nodules; NRH, nodular regenerative hyperplasia

\subsection{Treatment}

In the acute stage, all the patients received pulse methyl prednisolone and cyclophosphamide, in addition to anticoagulants with heparin. This was followed by the prescription of warfarin and azathioprine, these being general treatments for the condition. The patients were 

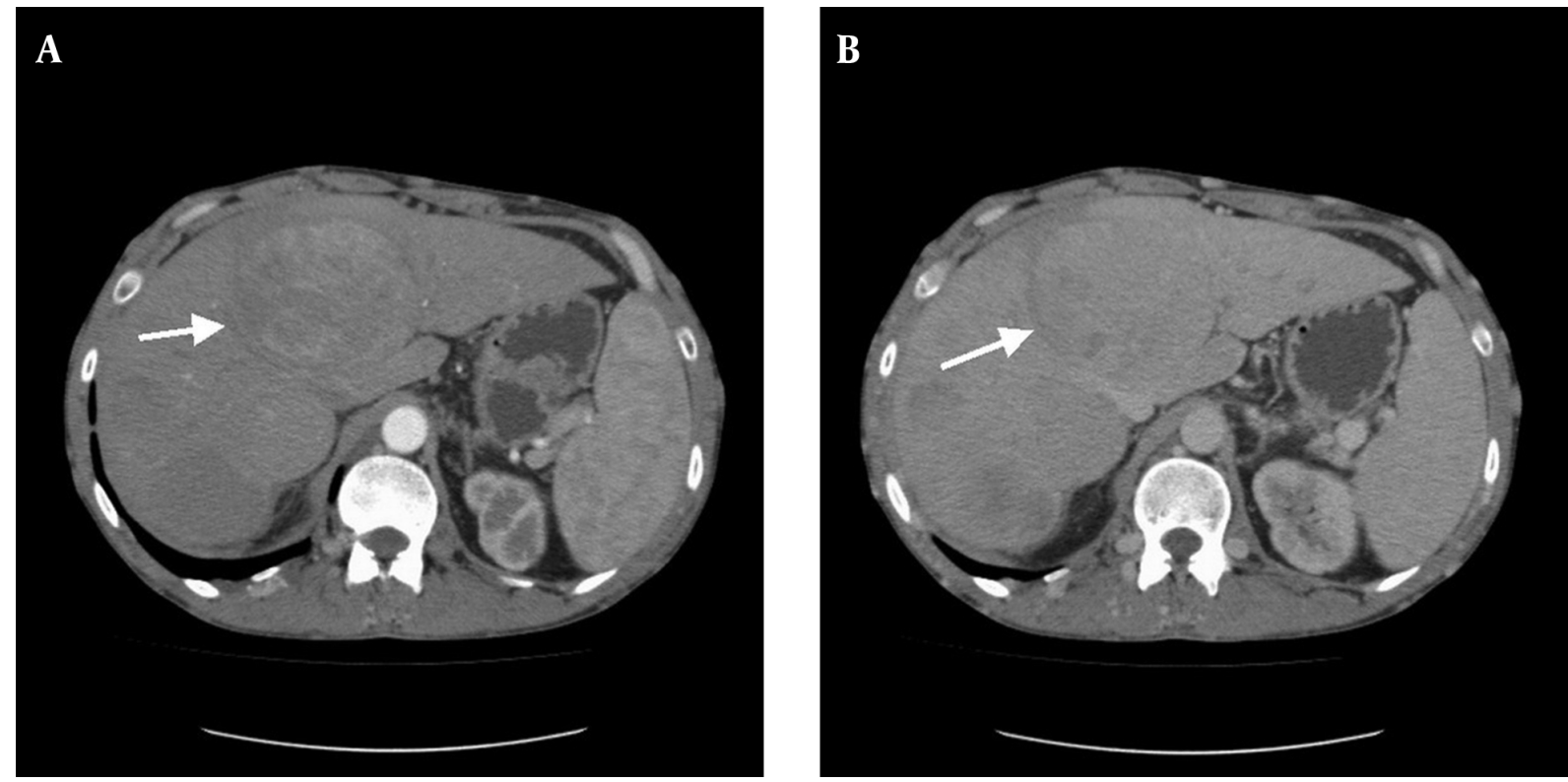

Figure 1. A, Large regenerative nodule of the liver (the largest one, with a diameter of $9 \times 8 \mathrm{~cm}$, has settled in segment $4 \mathrm{~A}-4 \mathrm{~B}$ ); B, CT imaging of the mass in venous phase (Case 3).
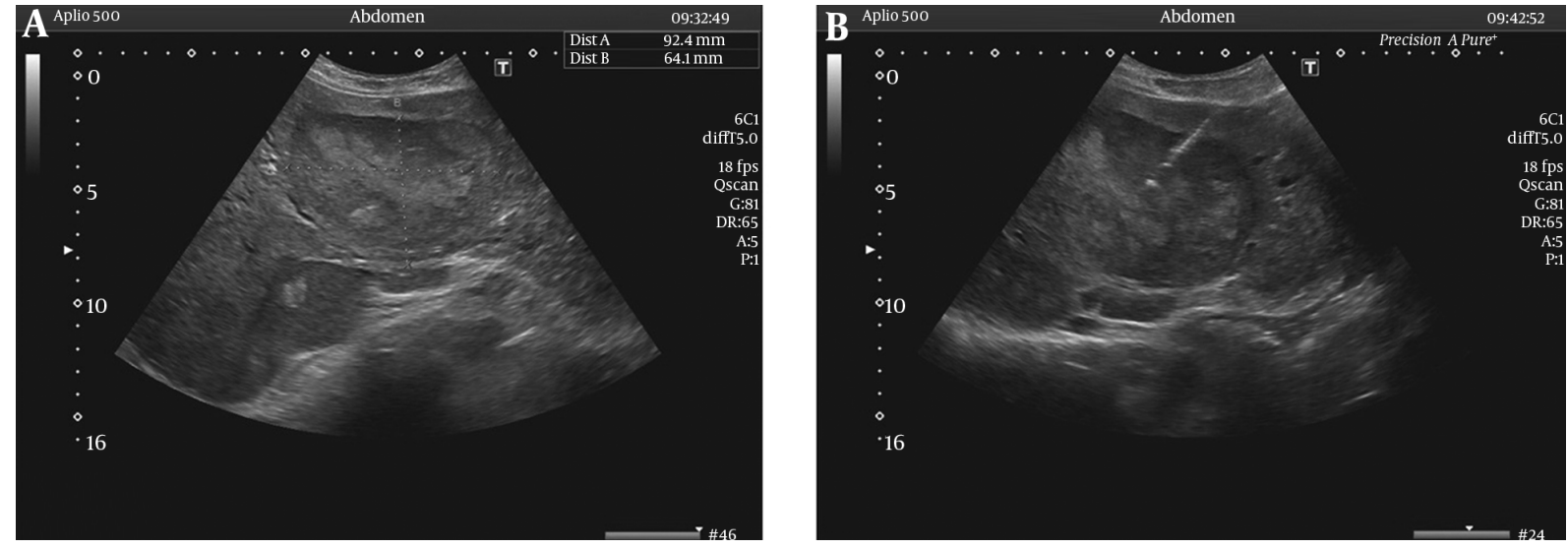

Figure 2. A, Large regenerative nodule of the liver via USG (the largest nodule, with a diameter of $9 \times 8 \mathrm{~cm}$, has settled in segment 4A-4B); B, USG-guided liver mass biopsy (Case 3).

evaluated for radiological or surgical interventions but, unfortunately, all patients with BCS caused by BD have ICV thrombosis, which results in an unsuitable pressure gradient occurring between the portal vein and the inferior vena cava, and so radiological interventions and portocaval shunt operations are technically impossible.

\section{Discussion}

The worldwide geographical distribution of primary and secondary BCS is highly variable, and there is scarcity of data in developing countries due to its rarity. The third most common reason for the occurrence of BCS in Turkey (where the incidence of BD is extremely high) is $\mathrm{BD}(4)$. The BCS occurrence rate during the course of BD was reported as being $0.35 \%$ in a study conducted in Turkey, in which the diagnosis of $\mathrm{BD}$ was made at the same time as the diagnosis of BCS in $71.4 \%$ of the patients (8). In our series, the BCS occurrence rate during the course of $\mathrm{BD}$ was $1.2 \%$, and the diagnosis of $\mathrm{BD}$ was made at the same time as the diagnosis of BCS in $40 \%$ of the patients. 
BCS results from occlusion of the three hepatic veins in about $2 / 3$ of cases, isolated occlusion of the IVC in about $10 \%$ of cases, and combined occlusion in almost $1 / 3$ of cases (9). Uskudar et al. and Harmanci et al. found there to be $30 \%$ combined venous occlusion in BD patients with BCS $(4,5)$. In our series, the combined venous occlusion rate was $100 \%$.

In a study by Bayraktar et al., which followed $14 \mathrm{BD}$ patients with BCS within a series of 493 BD patients (3\%), the authors concluded that the extent of vascular thrombosis within the inferior vena cava, rather than the presence of the hepatic vein thrombosis per se, was the major determinant of survival (10). Nowadays, in addition to the involvement of HVs and the VCI, PVT has come into prominence as a prognostic indicator in BD patients with BCS (4, $5,11,12)$. Harmanci et al. found that none of the aforementioned prognostic parameters (i.e., vascular involvement sites) were correlated with mortality, except for the presence of PVT (which had an estimated instantaneous risk of 8.4). They explain that PVT may indirectly reflect the degree of hepatic distress produced by outflow production due to the "hepatoportal venous reflex" (5). Darwish Murad et al. undertook a large international study, investigating patients diagnosed with nonmalignant BCS between 1984 and 2001 and classifying them into isolated BCS cases $(\mathrm{n}=$ 204), BCS-PVT cases without spleno-mesenteric vein thrombosis (SMVT; $\mathrm{n}=15$ ), and cases of BCS-PVT with SMVT ( $\mathrm{n}=$ 18). The survival rate after five years was $59 \%$ (95\% with a CI of $39 \%-80 \%$ ) in BCS-PVT, versus $85 \%$ (95\% with a CI of $76 \%$ $88 \%)$ in isolated BCS $(\mathrm{P}=0.11)(12)$. The data obtained from the literature on BCS patients reflects that the results come from heterogeneous, general patient groups. Further analysis reveals that PVT was present in just one of the eight BCS patients with BD in Harmanci et al.'s research $(\mathrm{P}=0.667)$ and in none of the nine BCS patients with BD in the study by Uskudar et al. Although both studies conclude that PVT is a negative factor in the prognosis for BCS generally, it was impossible to determine the effect of PVT upon BCS patients with BD specifically due to limited patient numbers $(4,5)$. In light of the worsening prognosis for BCS patients with PV involvement, it might be argued that determining the factors that can contribute to the development of PVT would be benefical in terms of seeking methods of prevention and treatment. Bagheri Lankarani et al. found, however, that there were no significant risk factors for PVT patients with cirrhosis awaiting liver transplantation in Shiraz, Iran (13). In our study, PVT was determined in one patient only, who died during the acute period due to ALF; the other patients, all of whom survived, tested negative for PVT. A study including large numbers of BCS patients with BD and demonstrating PVT would be helpful in determining the long-term prognostic significance of PVT in BCS patients with BD.

The survival time of patients after diagnosis of BCS can vary from between 3 months and 16 years, according to the literature (14). Our data, therefore, with a survival time of 1 month to 16 years after diagnosis of BCS, was consistent with the literature.

Hepatic masses might be detected during long-term follow-ups for BCS patients. To date, several studies from different regions have reported that a proportion of cases of BCS, especially in patients with VCI obstruction, are complicated by the development of hepatocellular carcinoma (HCC) in the long term $(14,15)$. As patients with HCC and concomitant hepatitis were excluded from these studies, the pooled prevalence of HCC in BCS was $15.4 \%$. The risk factors for HCC in BCS included the hepatic venous pressure gradient and being female (in two Asian studies), and factor $\mathrm{V}$ Leiden mutation, being male, and inferior vena cava obstruction (in one European study) (14). As is evident, there are huge differences between studies. The risk factors for HCC in BCS vary, depending on the geographical origin of the studies. As well as HCC, large regenerative nodules (LRNs) caused by outflow obstructions in the hepatic veins or vena cava are the masses most commonly associated with BCS (16). Nodular regenerative hyperplasia (NRH) and LRNs are distinct types of hepatocellular nodules. Indeed, the distinction between them is critical because their clinical significance is quite different. Ames et al. found that NRH was often associated with organ transplantation, myeloproliferative disease, and autoimmune processes. In their study, CT and MRI showed no enhancing liver masses in any of the patients with NRH. In contrast, LRNs were often associated with BCS (17). In accordance with the literature, hepatic masses were detected in 2 patients in this study through radiological surveillance and liver biopsies (LNRs in Case 3 and NRH with cirrhosis in Case 4).

Currently, clinical judgment and local expertise play important roles in the management of BCS. Medical therapy consists of the treatment of underlying disease, anticoagulation efforts, and symptom control. Emerging technologies have offered new minimally invasive treatment modalities, such as percutaneous catheter-directed thrombolysis, angioplasty, stenting, and TIPS (9). Although there is no controlled study in the existing literature regarding the benefits of immunosuppressive drug therapy for patients with BCS caused by BD, treatments used in the literature for BD with BCS include immunosuppressive and anticoagulation drugs, as well as the relief of hepatic venous outflow obstructions in order to preclude hepatocellular necrosis. Unfortunately, most patients with BCS caused by BD have inferior vena cava thrombosis, resulting in an unsuitable pressure gradient existing between 
the portal vein and the inferior vena cava, thus rendering a porto-caval shunt operation technically impossible. Another difficulty with managing these patients is that they usually have long-segment thrombosis, as well as thrombosis of other vascular structures (4). Therefore, medical treatment was approached to our patients.

Our study has several limitations. The most significant are the retrospective design and the small sample size, which make it impossible for us to generalize our results for clinical practice. Another limitation is the absence of a disease control group.

To conclude, as a result of the involvement of prominent vascular settlement sites in BD, BCS may develop. Although there are no exact data in the literature about vascular involvement sites as prognostic indicators in BCS patients with $\mathrm{BD}$, it has been demonstrated that portal venous thrombosis is prominent in general in BCS patient groups. Despite limited patient numbers, our data seems to support recent findings. In addition, the risk of cirrhosis and/or HCC development emerge during long-term follow-up investigations. Thus, the occurrence of cirrhosis and HCC must be taken into consideration as measures of surveillance, in addition to the presence of portal venous thrombosis. Furthermore, patients must be followed up at regular intervals via physical examinations, laboratory tests, and imaging procedures.

\section{Footnotes}

Authors' Contribution: Study concept and design: Dondu Uskudar Cansu and Cengiz Korkmaz; acquisition of data: Dondu Uskudar Cansu, Timucin Kasifoglu, Berat Acu, and Adem Erturk; analysis and interpretation of data: Dondu Uskudar Cansu and Temel; drafting of the manuscript: Dondu Uskudar Cansu; critical revision of the manuscript for important intellectual content: Dondu Uskudar Cansu, Cengiz Korkmaz and Tuncer Temel; statistical analysis: Dondu Uskudar Cansu; study supervision: Dondu Uskudar Cansu and Cengiz Korkmaz.

Conflicts of Interest: The authors declare no conflicts of interest.

Funding/Support: No funding or support received.

\section{References}

1. Kural-Seyahi E, Fresko I, Seyahi N, Ozyazgan Y, Mat C, Hamuryudan V, et al. The long-term mortality and morbidity of Behcet syndrome: a 2decade outcome survey of 387 patients followed at a dedicated center. Medicine (Baltimore). 2003;82(1):60-76. [PubMed: 12544711].
2. Ideguchi H, Suda A, Takeno M, Ueda A, Ohno S, Ishigatsubo Y. Behcet disease: evolution of clinical manifestations. Medicine (Baltimore). 2011;90(2):125-32. doi: 10.1097/MD.0b013e318211bf28. [PubMed: 21358436].

3. Valla DC. The diagnosis and management of the Budd-Chiari syndrome: consensus and controversies. Hepatology. 2003;38(4):793803. doi: 10.1053/jhep.2003.50415. [PubMed:14512865].

4. Uskudar O, Akdogan M, Sasmaz N, Yilmaz S, Tola M, Sahin B. Etiology and portal vein thrombosis in Budd-Chiari syndrome. World J Gastroenterol. 2008;14(18):2858-62. [PubMed:18473410].

5. Harmanci O, Kav T, Peynircioglu B, Buyukasik Y, Sokmensuer C, Bayraktar Y. Long-term follow-up study in Budd-Chiari syndrome: single-center experience in 22 years. J Clin Gastroenterol. 2013;47(8):706-12. doi: 10.1097/MCG.0b013e31824ffd63. [PubMed: 22495815].

6. Criteria for diagnosis of Behcet's disease. International Study Group for Behcet's Disease. Lancet. 1990;335(8697):1078-80. [PubMed: 1970380].

7. Bhakta B, Hamuryudan V, Brennan P, Chamberlain MA, Barnes C, Silman AJ. Assessment of disease activity in Behcet's disease. In: Wechsler B, Godeau P, editors. Excerpta medica international congress series 1037, 611. Amsterdam: Elsevier Science; 1993. pp. 235-40.

8. Tascilar K, Melikoglu M, Ugurlu S, Sut N, Caglar E, Yazici H. Vascular involvement in Behcet's syndrome: a retrospective analysis of associations and the time course. Rheumatology (Oxford). 2014;53(11):2018-22. doi: 10.1093/rheumatology/keu233. [PubMed: 24907156].

9. Horton JD, San Miguel FL, Membreno F, Wright F, Paima J, Foster $\mathrm{P}$, et al. Budd-Chiari syndrome: illustrated review of current management. Liver Int. 2008;28(4):455-66. doi: 10.1111/j.14783231.2008.01684.x. [PubMed: 18339072].

10. Bayraktar Y, Balkanci F, Bayraktar M, Calguneri M. Budd-Chiari syndrome: a common complication of Behcet's disease. Am J Gastroenterol. 1997;92(5):858-62. [PubMed: 9149201].

11. Mahmoud AE, Helmy AS, Billingham L, Elias E. Poor prognosis and limited therapeutic options in patients with Budd-Chiari syndrome and portal venous system thrombosis. Eur J Gastroenterol Hepatol. 1997;9(5):485-9. [PubMed: 9187882].

12. Darwish Murad S, Valla DC, de Groen PC, Zeitoun G, Haagsma EB, Kuipers EJ, et al. Pathogenesis and treatment of Budd-Chiari syndrome combined with portal vein thrombosis. Am J Gastroenterol. 2006;101(1):83-90. doi: 10.1111/j.1572-0241.2006.00353.x. [PubMed: 16405538].

13. Bagheri Lankarani K, Homayon K, Motevalli D, Heidari ST, Alavian SM, Malek-Hosseini SA. Risk Factors for Portal Vein Thrombosis in Patients With Cirrhosis Awaiting Liver Transplantation in Shiraz, Iran. Hepat Mon. 2015;15(12):ee26407. doi: 10.5812/hepatmon.26407. [PubMed: 26977162].

14. Ren W, Qi X, Yang Z, Han G, Fan D. Prevalence and risk factors of hepatocellular carcinoma in Budd-Chiari syndrome: a systematic review. Eur J Gastroenterol Hepatol. 2013;25(7):830-41. doi: 10.1097/MEG.0b013e32835eb8d4. [PubMed: 23411869].

15. Bayraktar Y, Egesel T, Saglam F, Balkanci F, Van Thiel DH. Does hepatic vein outflow obstruction contribute to the pathogenesis of hepatocellular carcinoma?. J Clin Gastroenterol. 1998;27(1):67-71. [PubMed: 9706774].

16. Tanaka M, Wanless IR. Pathology of the liver in Budd-Chiari syndrome: portal vein thrombosis and the histogenesis of veno-centric cirrhosis, veno-portal cirrhosis, and large regenerative nodules. Hepatology. 1998;27(2):488-96. doi: 10.1002/hep.510270224. [PubMed: 9462648].

17. Ames JT, Federle MP, Chopra K. Distinguishing clinical and imaging features of nodular regenerative hyperplasia and large regenerative nodules of the liver. Clin Radiol. 2009;64(12):1190-5. doi: 10.1016/j.crad.2009.07.015. [PubMed: 19913129]. 